\title{
STELLA Experiment: Hardware Issues
}

K.P. Kusche, L.P. Campbell, S.C. Gottschalk, W.D. Kimura,

D.C. Quimby, K.E. Robinson and L.C. Steinhauer

STI Optronics, Inc.

Bellevue, WA 98004

K.P. Kusche, M. Babzien, I. Ben-Zvi, J.C. Gallardo,

I.V. Pogorelsky, J. Skaritka, A. van Steenbergen and V. Yakimenko

National Synchrotron Light Source

Brookhaven National Laboratory

Upton, New York 11973

D.B. Cline, P. He and Y. Liu

UCLA

Los Angeles, CA 90024

R.B. Fiorito

Catholic University of America

Washington, DC 20064

R.H. Pantell

Stanford University

Stanford, CA 94305

\author{
D.W. Rule \\ Naval Surface Warfare Center \\ West Bethesda, MD 20817 \\ J. Sandweiss \\ Yale University \\ New Haven, CT 06520
}

July 1998

National Synchrotron Light Source

Brookhaven National Laboratory

Operated by

Brookhaven Science Associates

Upton, NY 11973

Under Contract with the United States Department of Energy

Contract Number DE-ACO2-98CH10886 


\section{DISCLAIMER}

This report was prepared as an account of work sponsored by an agency of the United States Govemment. Neither the United States Government nor any agency thereof, nor any of their employees, nor any of their contractors, subcontractors or their employees, makes any warranty, express or implied, or assumes any legal liability or responsibility for the accuracy, completeness, or any third party's use or the results of such use of any information, apparatus, product, or process disclosed, or represents that its use would not infringe privately owned rights. Reference herein to any specific commercial product, process, or service by trade name, trademark, manufacturer, or otherwise, does not necessary constitute or imply its endorsement, recommendation, or favoring by the United States Government or any agency thereof or its contractors or subcontractors. The views and opinions of authors expresses herein do not necessarily state to reflect those of the United States Government or any agency thereof. 


\title{
STELLA Experiment: Hardware Issues
}

\author{
K. P. Kusche, ${ }^{*}{ }^{\dagger}$ M. Babzien ${ }^{\dagger}$ I. Ben-Zvi, ${ }^{\dagger}$ L. P. Campbell,* D. B. Cline, ${ }^{\ddagger}$ \\ R. B. Fiorito, ${ }^{\S}$ J. C. Gallardo, ${ }^{\dagger}$ S. C. Gottschalk, ${ }^{*}$ P. He, ${ }^{\ddagger}$ W. D. Kimura, ${ }^{*}$ \\ Y. Liu, ${ }^{\ddagger}$ R. H. Pantell, ${ }^{\square}$ I. V. Pogorelsky, ${ }^{\dagger}$ D. C. Quimby, ${ }^{*}$ \\ K. E. Robinson, ${ }^{*}$ D. W. Rule, ${ }^{\#}$ J. Sandweiss, ${ }^{\forall}$ J. Skaritka, ${ }^{\dagger}$ \\ A. van Steenbergen, ${ }^{\dagger}$ L.C. Steinhauer, ${ }^{*}$ and V. Yakimenko ${ }^{\dagger}$ \\ *STI Optronics, Inc., Bellevue, WA 98004 \\ ${ }^{\dagger}$ Brookhaven National Laboratory, Upton, NY 11973 \\ ${ }^{\ddagger} U C L A$, Los Angeles, CA 90024 \\ ${ }^{\S}$ Catholic University of America, Washington DC 20064 \\ "Stanford University, Stanford, CA 94305 \\ ${ }^{\#}$ Naval Surface Warfare Center, West Bethesda, MD 20817 \\ ${ }^{\forall}$ Yale University, New Haven, CT 06520
}

\begin{abstract}
The STaged ELectron Laser Acceleration (STELLA) experiment is currently being assembled and tested at the BNL Accelerator Test Facility (ATF). The existing BNL inverse free electron laser (IFEL) has been positioned upstream of the inverse Cerenkov acceleration (ICA) experiment on Beamline \#1. This beamline also features new quadrupoles and a new spectrometer capable of $a+1-20 \%$ energy acceptance. A new laser beam transport system has been installed to permit accurate control of the laser phase for the laser beams sent to the IFEL and ICA devices. Detection of the microbunches will be performed using a coherent transition radiation (CTR) diagnostic similar to one already demonstrated at the ATF.
\end{abstract}

\section{INTRODUCTION}

The acceleration of electrons via laser interaction has been demonstrated recently by various experimental schemes, including the inverse Cerenkov acceleration (ICA) (1) and inverse free electron laser (IFEL) (2) experiments. These schemes featured the modulation of electron macrobunches over a short distance at a single interaction point, where the macrobunch length $(\mathrm{L} \sim 10 \mathrm{psec})$ greatly exceeded the input laser wavelength $(\lambda=10.6 \mu \mathrm{m})$. Acceleration was inefficient, as expected, as evidenced by 
a wide energy spectrum. Thus, for monochromaticity, it is desired to have $L<\lambda$. This can be accomplished by converting the macrobunch into a train of microbunches, where the microbunch length, $\ell$, is shorter than $\lambda$.

The arrangement described here proposes to accomplish this by staging a - microbunch-producing device in front of an accelerating section. Specifically, the IFEL wiggler, optimized for microbunching, will be utilized as a prebuncher for the ICA accelerating stage. Clearly, each of these sections has been proven to work independently of one other. But, such a combination presents a variety of engineering and hardware challenges, namely stabilizing and synchronizing each part of the experiment with respect to each other.

\section{DESIGN PHILOSOPHY}

In the early preparations for STELLA, it was determined that major modifications to the ATF beamline \#1 had to be performed; in fact, the entire beamline had to be rebuilt to meet the criteria of the project. This challenge presented certain optimizing opportunities that became practical due to the scope of the changes involved.

First, it was desirable to minimize the beamline assembly time in the experimental hall so as to maximize running time for all users of the facility. It was therefore decided to modularize the major sections of beamline \#1. The sections were designed to be assembled and presurveyed at a separate facility, and then installed at the ATF with minimum impact to other experiments. Another reason for modularity comes from the future proposed uses of beamline \#1, such as for the $\mathrm{FEL}^{2}$ and Compton Scattering experiments, where the section of beamline containing the ICA gas cell will be replaced by other interaction chambers.

A standardized low-profile rigid rail assembly was devised to locate and support all beamline components. This was done for several reasons. First, the rail system provides an extremely flexible component positioning method. The axial locations of many of the components, specifically the precise positions of the quadrupoles, had yet to be determined. The ability to reposition these elements was desirable. The rail system allows for the sliding of components into their desired axial locations with minimal need for survey and support modifications.

Standardized component clamping and the ability to presurvey the components to the rail and then to survey the rail onto the beamline was also desirable. This saved significant survey time. The geometry of beamline \#1 presented certain challenges, as well. Specifically, the distances from the beamline axis to various horizontal surfaces varied greatly, and the mounting of Chinese quadrupoles required a special design of the interface between magnet and rail. It was found that, due to manufacturing tolerances of the support points on the quadrupoles and other beamline components, adjustable interface points were necessary.

It was, therefore, decided that all components which were sensitive to $x, y$ alignment had to have an independently adjustable interface between the component 
and rail. Theoretically, once the component is presurveyed onto the beamline, it could be removed, serviced, replaced, and/or repositioned without the need for extensive resurvey. The rails were designed to kinematically locate onto the adjustable supports so that entire rail sections could be relocated with minimum survey.

The thermal stability of the laser (optical) and electron beamlines was evaluated for mechanical stabilization. The kinematic supports of the rails were configured so as to minimize the effects of random thermal excursions. The optical rails were located on the ceiling of the ATF experimental hall and anchored near where the bending mirrors are located. This philosophy was implemented to minimize the effect of thermal expansion on mirror alignment and stability, and took advantage of a unique feature of the hall. At the ATF, the entire overhead shielding structure of the experimental hall resides inside of a temperature-controlled building. Therefore, since the temperature is relatively stable on either side of the massive concrete roof beam, there is negligible flexing due to daily environmental changes. In short, the roof is as stable as the floor and can be safely used to support critical optical components and maintain precise alignment to components below.

The rail support system was analyzed and designed to be tolerant of thermal excursions. One example of this can be found in the supports for triplet-rail \#3. Since the two supports had to be different lengths, with one mounted to a concrete pedastal and the other to the floor, it was decided to make them from different materials. For the longer support, steel was chosen; for the shorter, aluminum. In this way, the pitch of the rail would not be expected to vary more than about $2 \mu \mathrm{m} / \pm 1^{\circ} \mathrm{C}$.

The rails and kinematic supports were analyzed for deflection and contact stresses. Beneath rails \#3\&4, the V-groove of the support system has two possible locations so as to minimize rail flexure in the event changes in the quadrupole size and/or position become necessary. All major electron beamline components have a 5-point support system incorporated into the rail interface. This provides ease of alignment as well as maximum rigidity.

Critical components such as the ICA gas cell, IFEL wiggler, and GPOP1 cross have axial anchoring such that they could theoretically be removed from the beamline, serviced, and replaced without the need for axial resurvey. The anchoring points are very near the cup/cone locator of the rail's kinematic support, so the thermal and mechanical effects on these critical components are minimized.

The two large crosses needed for laser beam incoupling (GPOP1 \& GPOP3) presented significant engineering challenges. Large in-vacuum mirrors have to be repositioned in a precise manner to assure laser beam alignment stability. Each is mounted to two parallel ground stainless steel rods, with support provided by a springclamped vee and flat interface. Air-actuated linear feedthroughs are used to position the mirrors into and away from the electron beam axis. An ATF-designed pop-in flag assembly is mounted to the same flange, but is controlled by a separate actuator. The flags have the capability of mounting either a standard phosphor-coated screen or a "YAG crystal" (3) in front of a feducialized $45^{\circ}$ outcoupling mirror. Such flags are necessary in order to visualize the e-beam profile at a distance no more than a few 
centimeters away from the hole in the center of the large incoupling mirror. They also allow for precise presurvey and feducialization of the cross assembly.

\section{CRITICAL ISSUES}

Ultimately, the ability to effectively synchronize the electron-laser interactions, upon which the success of the STELLA program relies, requires that several critical issues be addressed. Obviously, the ATF will be expected to deliver the $45 \mathrm{MeV}$ and 1 GW $\mathrm{CO}_{2}$ laser beam within specified parameters (4) that can be reasonably achieved. The STELLA project has taken responsibility for the creation and integration of systems to facilitate the transport of these beams to their respective interaction points (Figure 1). Comprehensive descriptions of the overall STELLA beamline functionalities $(4,5)$, as well as specific subsystem details regarding electron beam microbunch formation and detection $(4,6,7)$, are covered elsewhere. The critical issues discussed here include laser transport and phase stability, and spectrometry.

In order to enable high power (gigawatt regime) $\mathrm{CO}_{2}$ laser beam delivery to the IFEL and ICA interaction regions, several requirements must be met. A zinc selenide ( $\mathrm{ZnSe}$ ) beamsplitter is necessary to divert $<10 \%$ of the total laser power to the IFEL wiggler while allowing the remainder to be transmitted to the ICA gas cell. Due to the high peak power of each laser pulse $(\sim 100 \mathrm{psec})$, the beam must be sufficiently expanded to avoid optical damage; $<0.5 \mathrm{~J} / \mathrm{cm}^{2}$ for $\mathrm{ZnSe}$ lenses and windows, $<5 \mathrm{~J} / \mathrm{cm}^{2}$ for copper mirrors.

Delivery to the IFEL subsystem is achieved using axicon and spherical lens pairs to create an expanded-converging annular (central null) beam which is introduced into the vacuum beamline using the unique high-stability pop-in mirror (with central hole for e-beam passage) at the GPOP1 location. The annular beam is co-aligned with the electron beam as it forms a waist at the wiggler's sapphire waveguide, and is outcoupled at GPOP2 \& 3 for diagnostic purposes.

Delivery to the ICA subsystem can be handled by one of two optical schemes (denoted "old" \& "new") for converting the beam to the required radial polarization (RPC), after traversing the remotely-controllable delay rail path. Co-alignment of the axicon-focused laser beam to the triplet-focused e-beam at the gas cell has been previously facilitated via internal encoder-mike mirrors and pop-in screen. More stringent requirements for STELLA $(<100 \mu \mathrm{m}$ size regime for both beams) have forced a redesign of the methods by which both beams can be visualized simultaneously at high resolution. A working group has been formed to investigate the possibilities and begin testing at least one promising scheme.

The stated stability requirement for the laser beam path length (one leg with respect to the other) is $\pm 0.45 \mu \mathrm{m}$ (4). A stepper motor has been fitted to the delay rail to provide $\sim 0.2 \mu \mathrm{m}$ steps over a total distance of $>40 \mathrm{~cm}$, as confirmed using an LVDT measurement system. Stability studies have been performed to determine partial-path jitter and drifh, using a simple interferometer and $\mathbb{R}$ camera/frame grabber 
combination to visualize fringes from $632 \mathrm{~nm} \mathrm{HeNe}$ and $10 \mu \mathrm{m} \mathrm{CW} \mathrm{CO} 2$ lasers. Shotto-shot $(1 \mathrm{~Hz})$ jitter was found to be $\leq \pm 1 \mu \mathrm{m}$, but not better than $0.6 \mu \mathrm{m}$, and drift over $>0.5$ hours was $\leq+2 \mu \mathrm{m}$. As this configuration used temporary optical mounts without beam path enclosures, these numbers represent upper limits on the expected full-path stability. Our goals are likely to be met with the installation of new optical mounts and full beam path enclosures. Total beam path interferometric studies will follow soon after installation of these upgrades, which will determine the need for active feedback via piezostage, etc. Nonetheless, falling short of the stated jitter tolerance will not totally prevent bunch acceleration, just make it more difficult to observe.

The observation of the total energy-modulated spectrum will be accommodated by a versatile spectrometer, designed to meet the needs of the various stages of the STELLA program. At first, resolution on the order of $1 \%$ is desired for IFEL wiggler recommissioning; later, successful acceleration using the ICA subsystem requires up to $\pm 20 \%$ acceptance. The ensuing design incorporated such variable energy acceptance via $\pm 8^{\circ}$ horizontal rotation of a large dipole magnet, with vertical and horizontal beam size control by three quadrupoles and energy compensation by a sextupole. For ease of survey, as well as flexibility with other experiments, the same type of rail support system was implemented as for the rest of beamline \#1. A calculated energy resolution of up to $0.4 \%$ can easily accommodate all phases of STELLA. Imaging will initially be done with a standard phosphor screen and CCD/frame grabber system, as done in previous ICA experiments; an upgrade is planned and will be implemented if necessary. 


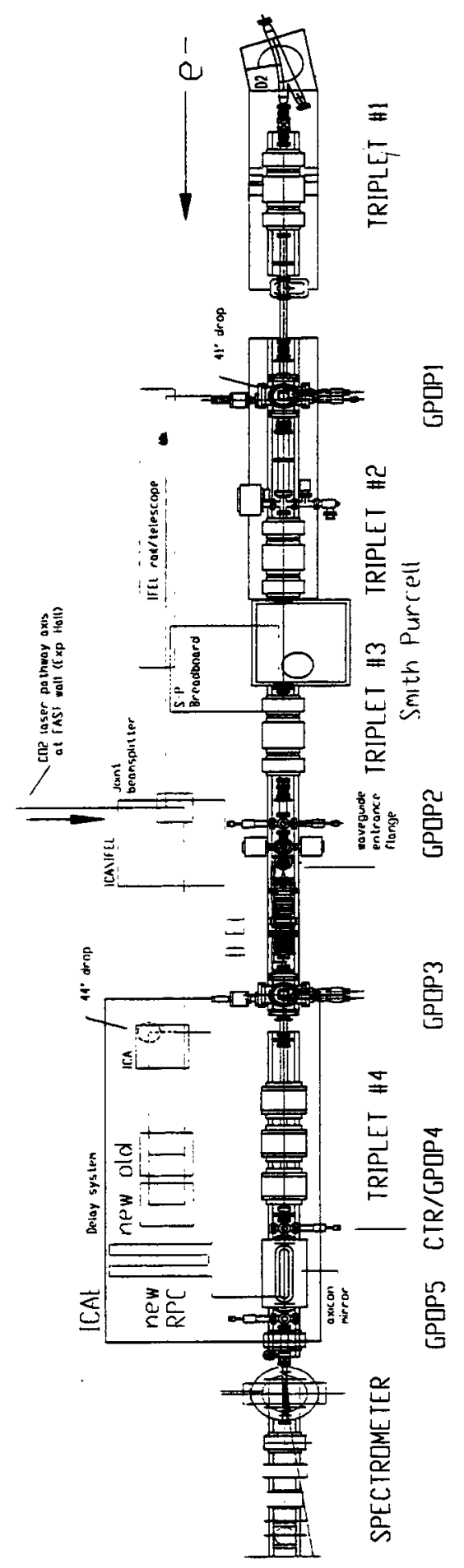

FIGURE 1. Actual layout for STELLA experiment on ATF beamline \#1. 


\section{CURRENT STATUS AND FUTURE PLANS}

All four of the quadrupole triplet-rail assemblies have been installed and commissioned at the ATF. Safety and operational approval has been granted for these subsystems as well as for the entire experiment. The upgrade of the laser transport system is in progress, with the goal of meeting the aforementioned stability requirements. Upon completion, total beam path interferometric studies are planned to quantify laser beam path jitter and drift. A measurement scheme, similar to that described for initial tests, will consist of a $\mathrm{CO}_{2}$ laser beam traversing both actual delivery paths terminating at a simple interferometer and $\mathbb{R}$ camera/analyzer combination to visualize the motion of 10.6 micron-spaced fringes.

The installation and commissioning of the spectrometer assembly is expected shortly. Coupled with successful delivery of laser power on the order of a few MW to the prebuncher, the spectrometer will enable characterization of the wiggler and CTR diagnostic. We expect to observe negligible electron beam quality degradation as it exits the wiggler, as well as evidence of microbunching at the CTR location and about $1 \%$ energy modulation at the spectrometer.

Only after these steps have been passed will the ICA gas cell be installed and commissioned. Low laser power $(<1 \mathrm{GW})$ delivery to both interaction regions will enable the optimization of the laser synchronization system, and will culminate in attempts to accelerate microbunches. Preliminary results from the fully-implemented STELLA configuration are expected in time for presentation at PAC'99. 


\section{CONCLUSION}

Comprehensive plans for the STELLA experiment are being implemented at the BNL ATF. Given the size of the collaboration, the luxury of having several working groups address critical issues in parallel has been exploited. Installation of key hardware is nearing completion, and preliminary results from electron and laser beam studies thus far indicate an absence of major hurdles toward the success of the experimental program.

\section{ACKNOWLEDGMENTS}

The authors wish to acknowledge the technical assistance of $\mathrm{R}$. Harrington, $\mathrm{L}$. Finch, X.J. Wang, and A. Doyuran, as well as the rest of the staff of the ATF, and the NSLS's survey, vacuum, and magnetic measurement groups. The long-standing support of the ATF and NSLS has been invaluable. This work was supported by the U.S. Department of Energy, Grant Nos. DE-FG03-98ER41061, DE-AC0298CH10886, and DE-FG03-92ER40695.

\section{REFERENCES}

1. Kimura, W. D., Kim, G. H., Romea, R. D., Steinhauer, L. C., Pogorelsky, I. V., Kusche, K. P., Fernow, R. C., Wang, X. J., and Liu, Y., Phys. Rev. Lett. 74, 546-549 (1995).

2. van Steenbergen, A., Gallardo, J., Sandweiss, J., Fang, J.-M., Babzien, M., Qiu, X., Skaritka, J., and Wang, X.J., Phys. Rev. Lett. 77, 2690 (1996).

3. Graves, W. S., Johnson, E. D., O'Shea, P. G., “A High Resolution Electron Beam Profile Monitor," Proceedings of the IEEE Particle Accelerator Conference, May 12-16, 1997, Vancouver, Canada; also, BNL Publication No. BNL-65006.

4. See Staged Electron Laser Acceleration (STELLA) Technical Proposal submitted to U.S. Department of Energy by STI Optronics, Inc., June 27, 1998.

5. Kimura, W. D., Babzien, M., Ben-Zvi, I., Campbell, L. J., Cline, D. B., Fiorito, R. B., Gallardo, J. C., Gottschalk, S. C., He, P., Kusche, K. P., Liu, Y., Pantell, R. H., Pogorelsky, I. V., Quimby, D. C., Robinson, K. E., Rule, D. W., Sandweiss, J., Skaritka, J., Steinhauer, L. C., van Steenbergen, A., and Yakimenko, V., "STELLA Experiment - Design and Model Predictions," in these Proceedings of the 8th Workshop on Advanced Accelerator Concepts, Jul. 5-11, 1998, Baltimore, MD.

6. Liu, Y., Cline, D. B., Wang, X. J., Babzien, M., Fang, J. M., Kusche, K.P., 1997 Particle Accelerator Conference, May 12-16, 1997, Vancouver, B.C., Canada, Paper 3'C3.

7. He, P., Babzien, M., Ben-Zvi, I., Campbell, L. J., Cline, D. B., Fiorito, R B., Gallardo, J. C., Gottschalk, S. C., Kimura, W. D., Kusche, K. P., Liu, Y., Pantell, R. H., Pogorelsky, I. V., Quimby, D. C., Robinson, K. E., Rule, D. W., Sandweiss, J., Skaritka, J., Steinhauer, L. C., van Steenbergen, A., and Yakimenko, V., "STELLA Experiment - Microbunch Diagnostic," in these Proceedings of the 8th Workshop on Advanced Accelerator Concepts, Jul. 5-11, 1998, Baltimore, MD. 